\title{
ESTUDO FITOQUíMICO E AVALIAÇÃO DA ATIVIDADE ANTI-INFLAMATÓRIA DE Aeschynomene fluminensis Vell. (Fabaceae) ${ }^{\#}$
}

\author{
Marlene Capelin Ignoato, Rodrigo Monteiro Fabrão, Ivânia Teresinha Albrecht Schuquel, Marcos Felipe Pinatto Botelho \\ e Silvana Maria de Oliveira Santin* \\ Departamento de Química, Universidade Estadual de Maringá, 87020-900 Maringá - PR, Brasil \\ Laura Lícia Milani de Arruda e Ciomar Aparecida Bersani-Amado \\ Departamento de Farmacologia e Terapêutica, Universidade Estadual de Maringá, 87020-900 Maringá - PR, Brasil \\ Maria Conceição de Souza \\ Departamento de Biologia, Universidade Estadual de Maringá, 87020-900 Maringá - PR, Brasil
}

Recebido em 11/12/11; aceito em 2/4/12; publicado na web em 2/7/12

\begin{abstract}
PHYTOCHEMICAL STUDY AND EVALUATION OF ANTI-INFLAMMATORY ACTIVITY OF Aeschynomene fluminensis Vell. (Fabaceae). Phytochemical investigation of Aeschynomene fluminensis leaves and branches led to isolation of the flavonoid glycosides kaempferol 3,7-di- $O$ - $\alpha$-L-rhamnopyranoside, kaempferol 7-O- $\alpha$-L-rhamnopyranoside, kaempferol 3-O-apiofuranosil7-O- rhamnopyranoside, quercitin 3-O- $\alpha$-L-rhamnopyranoside, quercitin 3-O-arabinofuranoside, 8- $\beta$-D-glucopyranosyl 4',5,7-trihydroxyflavanone, the isoflavonoid 4',7-di-hydroxy-isoflavone, the dimer epicatechin- $(2 \beta \rightarrow 7,4 \beta \rightarrow 8)$ - epicatechin, the polyol 3-O-methyl-chiro-inositol and two steroids in sitosterol and stigmasterol mixture. These compounds were identified by NMR ${ }^{1} \mathrm{H}$ and ${ }^{13} \mathrm{C}$ and compared with literature data. Anti-inflammatory activity of the crude methanolic extract and its fractions was evaluated.
\end{abstract}

Keywords: Aeschynomene fluminensis; flavonoides; anti-inflammatory activity.

\section{INTRODUÇÃO}

Espécies nativas do alto do rio Paraná, região de Porto Rico-PR, têm sido alvo de nossos estudos em conjunto com pesquisadores das áreas de botânica e de farmacologia da Universidade Estadual de Maringá. Esta área, denominada planície alagável, abrange parte da Área de Proteção Ambiental das Ilhas e Várzeas do Rio Paraná (PR e MS) e do Parque Estadual do Ivinhema (MS) - local considerado reserva ecológica pelo Estado. Nossos estudos visam a avaliação dos potenciais químico e farmacológico das espécies vegetais desta região.

O gênero Aeschynomene, pertencente à família Fabaceae, é representado por 52 espécies brasileiras, de um total de 160 espécies descritas com ocorrência na América, Ásia e África. Destas espécies, 84 se distribuem pelo continente americano, predominantemente na região neotrópica. ${ }^{1}$ Em levantamento bibliográfico para o gênero foram encontrados estudos químicos e/ou biológicos de apenas cinco espécies: A. indica, A. mimosifolia, A. virgata, A. sensitiva e A. stolzii. ${ }^{2}$ Estes estudos retratam a presença de flavonoides, neoflavonoides, isoflavonoides e rotenoides e, ainda, as atividades espermicida, inseticida, citotóxica e antitumoral para as espécies deste gênero. ${ }^{2}$

A espécie Aeschynomene fluminensis, conhecida popularmente como "cortiça", se desenvolve em regiões que são sazonalmente inundadas, ocorrendo no Brasil nos estados do Espírito Santo, Rio de Janeiro, Minas Gerais, Pará, Paraná e Mato Grosso do Sul. ${ }^{1}$

Neste trabalho são apresentados os resultados do estudo fitoquímico das folhas e dos galhos da espécie Aeschynomene fluminensis e da atividade anti-inflamatória dos extratos brutos e de suas frações.

*e-mail: smoliveira@uem.br

\#Artigo em homenagem ao Prof. Otto R. Gottlieb (31/8/1920-19/6/2011)

\section{PARTE EXPERIMENTAL}

\section{Procedimentos gerais}

Os espectros de RMN (uni- e bidimensionais) foram obtidos em espectrômetro Varian, modelo Mercury plus BB, operando a $300 \mathrm{MHz}$ para ${ }^{1} \mathrm{H}$ e 75,5 MHz para ${ }^{13} \mathrm{C}$. Os deslocamentos químicos foram dados em ppm, tendo como referência interna o sinal do solvente e/ou do tetrametilsilano (TMS, $\delta=0,0 \mathrm{ppm}$ ). Os solventes utilizados foram $\mathrm{CD}_{3} \mathrm{OD}$, e $\mathrm{CDCl}_{3}$ (Aldrich). Para as cromatografias em coluna (CC) utilizou-se gel de sílica 60 (0,063-0,200 mm, Merck) ou Sephadex LH-20 (Sigma), como fase estacionária. Para as cromatografias em camada delgada (CCD) empregou-se gel de sílica $60 \mathrm{G}$ e gel de sílica $60 \mathrm{GF}_{254}$ (Merck). A visualização das substâncias em CCD foi realizada por irradiação com luz ultravioleta em 254 e $366 \mathrm{~nm}$ e/ou por pulverização com solução de $\mathrm{H}_{2} \mathrm{SO}_{4}$ /anisaldeído/AcOH $(1: 0,5: 48,5)$ seguida de aquecimento.

\section{Material vegetal}

A planta Aeschynomene fluminensis foi coletada em março de 2008, às margens da bacia de inundação do alto do Rio Paraná, na região de Porto Rico-PR, divisa com o município de Baitaporã-MS. A exsicata do material vegetal encontra-se depositada no Herbário da Universidade Estadual de Maringá sob o registro n ${ }^{\circ}$ HUEM 15708.

\section{Isolamento dos constituintes químicos}

As folhas $(454,4 \mathrm{~g})$ e os galhos $(560,7 \mathrm{~g})$ de A. fluminensis foram secos ao ar, triturados e submetidos à extração exaustiva com metanol a frio, fornecendo 46,8 e 23,5 g de extratos brutos EBF e EBG, respectivamente. Partes destes extratos (20,0 g de cada) foram dissolvidas em $\mathrm{H}_{2} \mathrm{O}$ e particionadas em solventes de polaridade crescente, resultando nas frações hexânicas (FHF: 6,5 g e FHG: 1,3 g), clorofórmicas (FCF: 0,7 g e FCG: 0,9 g), acetato de etila (FAEF: 0,9 g 
e FAEG: 1,8 g), $n$-butanólicas (FBF: 9,5 g e FBG: 6,3 g) e aquosas (FAF: 1,5 g e FAG: 8,6 g) das folhas e dos galhos, respectivamente. A fração $\operatorname{FAEF}(0,28 \mathrm{~g})$ foi submetida a uma cromatografia em Sephadex LH-20 eluída com $\mathrm{MeOH} / \mathrm{H}_{2} \mathrm{O} 1: 1(\theta=1,5 \mathrm{~cm}$ e $5 \mathrm{~g})$, resultando em 130 frações de $2 \mathrm{~mL}$ cada, que foram reagrupadas após análise em CCD. As subfrações 29-32, 35-67, 71-80, 107-112 e 117-119 e 122-128 resultaram no isolamento das substâncias $\mathbf{1}$ (5,5 mg), 2 (5,8 mg), $\mathbf{5}(8,2 \mathrm{mg}), \mathbf{8}(4,7 \mathrm{mg})$ e das misturas de $\mathbf{1}$ e $\mathbf{3}$ $(15,4 \mathrm{mg})$ e de 1 e 7 (52,3 mg). A subfração 98-106 (30,9 mg) foi submetida a uma filtração em Sephadex LH-20 com MeOH $(\theta=1,0$ $\mathrm{cm}$ e $3 \mathrm{~g})$ resultando no isolamento da substância $4(6,0 \mathrm{mg})$. As frações FBF $(0,29 \mathrm{~g})$ e FAF $(0,49 \mathrm{mg})$ foram submetidas ao mesmo tratamento realizado para FAEF e forneceram novamente a substância $1(33,2 \mathrm{mg})$ e a mistura de 1 e $7(11,2 \mathrm{mg})$ para a primeira fração, e a substância $9(22,7 \mathrm{mg})$ para a segunda. A fração FAEG $(0,48 \mathrm{~g})$ foi submetida a uma cromatografia em gel de Sephadex LH-20 com $\mathrm{MeOH} / \mathrm{H}_{2} \mathrm{O}$, fornecendo a mesma mistura 1 e 7 (15,3 mg). Este mesmo tratamento foi realizado para a $\mathrm{FBG}(0,46 \mathrm{~g})$ e possibilitou o isolamento novamente da substância $\mathbf{1}(5,5 \mathrm{mg})$. Para a fração FAG $(0,58 \mathrm{~g})$ este tratamento resultou no isolamento da substância 9 (3,5 mg). Parte da FCG $(0,60 \mathrm{~g})$ foi fracionada em CC de gel de sílica $(\theta=2,0 \mathrm{~cm}$ e $21 \mathrm{~g})$ eluída com hexano e AcOEt em ordem crescente de polaridade hexano; hexano:AcOEt 10-95\% e AcOEt. A subfração hexano:AcOEt 50\% forneceu a substância $\mathbf{6}$ (4,4 mg). Parte da FHF $(0,34 \mathrm{~g})$ foi fracionada em CC de gel de sílica $(\theta=1,5 \mathrm{~cm}$ e $13 \mathrm{~g})$ eluída com misturas de hexano e AcOEt em ordem crescente de polaridade. A subfração hexano:AcOEt 15\% (3,9 mg) forneceu a mistura dos esteroides sitosterol e estigmasterol. O mesmo tratamento foi realizado para a FHG $(0,75 \mathrm{~g})$, sendo que a subfração hexano: AcOEt $20 \%(25,0 \mathrm{mg})$ forneceu a mesma mistura de esteroides.

\section{Avaliação da atividade anti-inflamatória}

A atividade anti-inflamatória foi avaliada pelo modelo de edema de orelha induzido por óleo de cróton, ${ }^{3}$ em camundongos Swiss machos (25-30 g). O edema de orelha foi induzido pela aplicação de 20 $\mu \mathrm{L}$ de solução óleo de cróton ( $200 \mu \mathrm{g}$ ) diluído em $10 \mathrm{~mL}$ de acetona/ água (7:3) na superfície interna da orelha esquerda do camundongo. Imediatamente após a aplicação do agente flogístico, nos grupos de animais tratados, foram aplicados $20 \mu \mathrm{L}$ de uma solução dos extratos brutos das folhas e dos galhos ou das suas frações de A. fluminensis, na superfície interna da orelha esquerda ( $5 \mathrm{mg} /$ orelha). A orelha direita recebeu apenas o veículo $(20 \mu \mathrm{L})$. Após $6 \mathrm{~h}$, os animais foram anestesiados, sacrificados e as orelhas seccionadas em discos de $6,0 \mathrm{~mm}$ de diâmetro. Estas secções foram pesadas em uma balança analítica. Utilizou-se a indometacina como controle positivo. Os resultados foram expressos como média \pm erro padrão da média (epm) e analisados utilizando teste de Student para comparação de duas médias, ou análise de variância (ANOVA) para múltiplas comparações, sendo utilizados $\mathrm{P}<0,05$ como nível de significância. A porcentagem da inibição do edema foi determinada pela Equação 1:

$$
(\%) \text { de inibição }=\frac{\begin{array}{c}
\text { peso das orelhas inflamadas }(\mathrm{OC}+\mathrm{V})- \\
\text { peso das orelhas tratadas }
\end{array}}{\begin{array}{c}
\text { peso das orelhas inflamadas }(\mathrm{OC}+\mathrm{V})- \\
\text { peso da orelha não inflamada }(\mathrm{V})
\end{array}} \times 100
$$

\section{Avaliação da atividade da mieloperoxidase (MPO)}

A atividade da MPO foi avaliada através do sobrenadante de homogenados das secções de orelhas direita (controles) e esquerda (tratadas com extrato bruto e frações, 5,0 mg e indometacina $1 \mathrm{mg}$ ), de acordo com a técnica descrita por Bradley. ${ }^{4} \mathrm{O}$ tecido da orelha foi colocado em tampão fosfato de potássio $50 \mathrm{mM}, \mathrm{pH} 6,0$, contendo
0,5\% de brometo de hexadeciltrimetil-amônio (Sigma - $1 \mathrm{~mL} / 50 \mathrm{mg}$ de tecido) em homogeneizador de Potter. O homogenado foi agitado em vórtex e centrifugado durante $5,0 \mathrm{~min}$ a $2500 \mathrm{rpm}$ e $25^{\circ} \mathrm{C}$. Foram adicionados $10 \mu \mathrm{L}$ do sobrenadante obtido em microplaca de 96 cavidades, em triplicata sendo, a seguir, adicionados $200 \mu \mathrm{L}$ de uma solução tampão contendo o-dianisidina di-hidrocloreto (Sigma-16,7 $\mathrm{mg})$, água bidestilada (90 mL), tampão fosfato de potássio $(10 \mathrm{~mL})$ e $\mathrm{H}_{2} \mathrm{O}_{2} 1 \%(50 \mu \mathrm{L})$. A reação foi interrompida pela adição de 30 $\mu \mathrm{L}$ de acetato de sódio $1,46 \mathrm{M}(\mathrm{pH} 3,0)$ e a atividade da enzima foi determinada pela técnica do ponto final, ${ }^{4}$ através de medida de absorbância $(450 \mathrm{~nm})$. A porcentagem de redução na atividade da enzima mieloperoxidase foi determinada pela Equação 1.

\section{RESULTADOS E DISCUSSÃO}

O estudo fitoquímico das folhas e galhos de A. fluminensis resultou no isolamento dos flavonoides 3,7-di- $O$ - $\alpha$-L-raminopiranosilcanferol (canferitrina) (1), 7- $O$ - $\alpha$-L-raminopiranosilcanferol ( $\alpha$-raminoisorobina) (2), 3-O-apiofuranosil-7- $O$ - $\alpha$-L-raminopiranosilcanferol (3), 3- $O$ - $\alpha$-L-raminopiranosilquercetina (quercitrina) (4), 3- $O-\alpha-\mathrm{L}-$ arabinofuranosilquercetina (avicularina) (5), o isoflavonoide 4',7-di-hidroxi-isoflavona (daidzeína) (6), a flavanona 8-C- $\beta$-Dglicopiranosil-4',5,7-tri-hidroxiflavanona (iso-hemifloina) (7), o dímero epicatequina- $(2 \beta \rightarrow 7,4 \beta \rightarrow 8)$-epicatequina (procianidina $\mathrm{A}_{2}$ ) (8), o poliol 3-O-metil-quiro-inositol (9). As estruturas das substâncias isoladas foram estabelecidas com base nas análises dos dados espectroscópicos (RMN ${ }^{1} \mathrm{He} \mathrm{e}^{13} \mathrm{C}$, DEPT, gCOSY ${ }^{1} \mathrm{H}-{ }^{1} \mathrm{H}$, gHSQC, gHMBC, NOESY) e por comparação com os dados disponíveis na literatura.<smiles>[R20]Oc1c(-c2ccc(O)c([R3])c2)oc2cc([R20])cc(O)c2c1=O</smiles>

$1 \mathrm{R}_{1}=\mathrm{R}_{2}=\mathrm{Rha}, \mathrm{R}_{3}=\mathrm{H}$ $2 \mathrm{R}_{1}=\mathrm{Rha}, \mathrm{R}_{2}=\mathrm{R}_{3}=\mathrm{H}$ $3 \mathrm{R}_{1}=\mathrm{Rha}, \mathrm{R}_{2}=\mathrm{Api}, \mathrm{R}_{3}=\mathrm{H}$ $4 \mathrm{R}_{1}=\mathrm{H}, \mathrm{R}_{2}=\mathrm{Rha}, \mathrm{R}_{3}=\mathrm{OH}$ $5 \mathrm{R}_{1}=\mathrm{H}, \mathrm{R}_{2}=\mathrm{Ara}, \mathrm{R}_{3}=\mathrm{OH}$

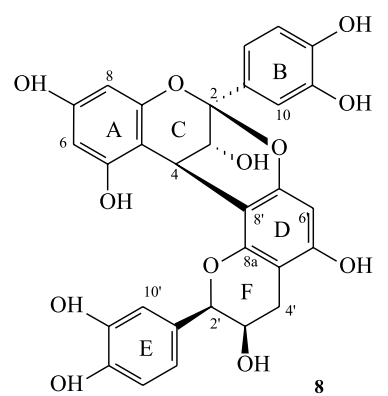<smiles>O=C1C[C@@H](c2ccc(O)cc2)Oc2c(O)c(O)cc(O)c21</smiles>

$\mathrm{HO}$
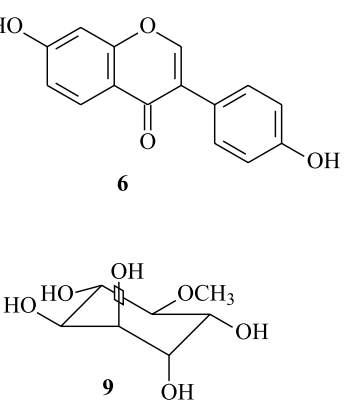

Figura 1. Estruturas dos metabólitos secundários de Aeschynomene fluminensis

A substância 1 foi obtida das frações acetato de etila e $n$-butanólica das folhas e dos galhos como sólido amorfo amarelo. Através do espectro de $\mathrm{RMN}$ de ${ }^{1} \mathrm{H}$ foram observados dois dupletos em $\delta_{\mathrm{H}} 7,89(\mathrm{~J}$ $=8,7 \mathrm{~Hz}, \mathrm{H}-2$ ' e H-6') e 6,93 ( $J=8,7 \mathrm{~Hz}, \mathrm{H}-3$ ' e H-5') característico de um anel $\mathbf{B} p$-hidroxilado, e de dois dupletos em $\delta_{\mathrm{H}} 6,46(J=1,8 \mathrm{~Hz}$, H-6), $6,72(\mathrm{~J}=1,8 \mathrm{~Hz}, \mathrm{H}-8)$ correspondentes ao anel $\mathbf{A}$, que indicaram como aglicona a estrutura 3,4',5,7-tetraidroxiflavona (canferol). A presença dos sinais na região de hidrogênios carbinólicos, em $\delta_{\mathrm{H}} 5,39$ ( $d, J=1,8 \mathrm{~Hz}, \mathrm{H}-1 ")$ e 5,56 ( $\left.s l, \mathrm{H}-1^{\prime \prime \prime}\right)$, característicos de hidrogênios 
anoméricos e dos sinais em $0,92(d ; J=5,7 \mathrm{~Hz}, \mathrm{H}-6 ")$ e $1,25(d ; J=$ $6,0 \mathrm{~Hz}, \mathrm{H}-6$ '") determinaram a presença de duas unidades de raminopiranose. Pelo espectro de $\mathrm{RMN}{ }^{13} \mathrm{C}$ foram observados sinais em $\delta_{\mathrm{C}} 95,6(\mathrm{C}-8), 100,6$ (C-6), 116,6 (C-3' e C-5'), 132,0 (C-2' e C-6') e 179,8 característicos da unidade de canferol, sinais em $\delta_{C} 103,5$ e 99,9 de carbonos anoméricos e de dois carbonos metílicos em $\delta_{\mathrm{C}} 17,7$ e 18,1, confirmando a presença de duas unidades de raminopiranose. Estas unidades foram inferidas nas posições C-3 e C-7 baseando-se nos deslocamentos químicos dos hidrogênios anoméricos em $\delta_{\mathrm{H}} 5,39$ (d, 1,8 Hz, H-1") e $\delta_{\mathrm{H}} 5,56$ (sl, H-1'") compatíveis a estas localizações quando comparados com aqueles encontrados na literatura, ${ }^{5}$ e ainda confirmadas pelo mapa de contornos HMBC pelas interações heteronucleares destes hidrogênios com os carbonos em $\delta_{\mathrm{C}} 136,5(\mathrm{C}-3)$ e 163,6 (C-7) da aglicona. A comparação dos dados espectroscópicos obtidos com os da literatura ${ }^{5}$ permitiu identificar a substância 1 como 3,7-di- $O$ - $\alpha$-L-raminopiranosilcanferol (canferitrina).

Nos espectros de RMN ${ }^{1} \mathrm{H}$ e ${ }^{13} \mathrm{C}$ das substâncias $\mathbf{2}$ e $\mathbf{3}$ foram observados sinais similares aos da substância $\mathbf{1}$, característicos de uma unidade canferol como aglicona e de uma raminopiranose na posição C-7. Para a substância 3 foram observados sinais adicionais em $\delta_{\mathrm{H}} 5,71$ $(d, J=1,8 \mathrm{~Hz}, \mathrm{H}-1 ") / \delta_{\mathrm{C}} 111,1(\mathrm{C}-1 ")$ característicos de anomérico, em $\delta_{\mathrm{H}} 3,60(m, \mathrm{H}-4 ") / \delta_{\mathrm{C}} 76,6(\mathrm{C}-4 ")$ e $3,53(\mathrm{~s}, \mathrm{H}-5 ") / \delta_{\mathrm{C}} 65,2\left(\mathrm{C}-5^{\prime \prime}\right)$ característicos de metilênicos e, ainda, os sinais $\delta_{\mathrm{H}} 4,28(d, J=1,8$ $\mathrm{Hz}, \mathrm{H}-2 ") / \delta_{\mathrm{C}} 79,2$ (C-2") de oximetínico e em $\delta_{\mathrm{C}} 81,1$ (C) de carbono não ligado a hidrogênio, que possibilitaram a identificação de uma unidade apiose. Os dados espectroscópicos de 2 e 3 foram comparados aos da literatura ${ }^{6}$ e permitiram definir as estruturas destes glicosídeos como 7- $O$ - $\alpha$-L-raminopiranosilcanferol ( $\alpha$-raminoisorobina) e 3-O-apiofuranosil-7- $O$-raminopiranosil-canferol.

A unidade aglicona dos glicosídeos $\mathbf{4}$ e $\mathbf{5}$ foi caracterizada como quercetina, através da análise dos espectros de $\mathrm{RMN}{ }^{1} \mathrm{H}$, pela presença dos sinais de um sistema $\mathrm{AB}$ atribuídos aos hidrogênios $\mathrm{H}-6 / \mathrm{H}-8\left[\delta_{\mathrm{H}}\right.$ $6,19 / 6,36, d, J=1,8 \mathrm{~Hz}(4)$ e $\left.\delta_{\mathrm{H}} 6,20 / 6,39, d, J=2,1 \mathrm{~Hz}(5)\right]$ e dos sinais típicos de anel B de flavonoides 3',4'-dissubstituídos, os hidrogênios $\mathrm{H}^{\prime} / \mathrm{H}^{\prime} / \mathrm{H}^{\prime}\left[\delta_{\mathrm{H}} 7,32, d, J=2,1 \mathrm{~Hz} / 6,90, d, J=8,1 \mathrm{~Hz} / 7,29, d d, J=\right.$ 8,1 e $2,1 \mathrm{~Hz}(4)$ e $\delta_{\mathrm{H}} 7,52, d, J=2,1 \mathrm{~Hz} / 6,89, d, J=8,4 \mathrm{~Hz} / 7,49$, $d d, J=8,4$ e $2,1 \mathrm{~Hz}(5)]$. A natureza glicosídica para as substâncias foi caracterizada pelos demais sinais oximetínicos e oximetilênicos observados nos espectros de $\mathrm{RMN}{ }^{1} \mathrm{H}$ e ${ }^{13} \mathrm{C}$. Para a substância 4, os sinais em $\delta_{\mathrm{H}} 5,33(d, J=1,5 \mathrm{~Hz}) / \delta_{\mathrm{C}} 103,5$ característico de $\mathrm{H} / \mathrm{C}$ anoméricos, e de metílicos em $\delta_{\mathrm{H}} 0,93(d, J=5,7 \mathrm{~Hz}) / \delta_{\mathrm{C}} 17,6$ indicaram a presença de uma raminose. A posição desta unidade no C-3 da quercetina foi confirmada pelo mapa de contornos HMBC, pela correlação heteronuclear deste carbono, em $\delta_{\mathrm{C}} 136,2$, com o hidrogênio anomérico em $\delta_{\mathrm{H}} 5,33$ (H-1"). Os dados espectroscópicos de 4 foram comparados com os da literatura ${ }^{7}$ sendo condizentes para 3-O- $\alpha$-L-raminopiranosil-quercetina (quercitrina). Para a substância 5, a presença dos sinais em $\delta_{\mathrm{H}} 5,46(d, J=0,9 \mathrm{~Hz}, \mathrm{H}-1 ") / \delta_{\mathrm{C}} 109,6$ $(\mathrm{C}-1 ")$ e $\delta_{\mathrm{H}} 3,49(d, J=4,8 \mathrm{~Hz}, \mathrm{H}-5 ") / \delta_{\mathrm{C}} 62,6$ (C-5") característicos de anoméricos e metilênicos, e os sinais $\delta_{\mathrm{H}} 4,32(d d, J=0,9$ e 3,0 $\mathrm{Hz}, \mathrm{H}-2 ") / \delta_{\mathrm{C}} 83,5(\mathrm{C}-2 "), 3,90(d d, J=3,0$ e $5,1 \mathrm{~Hz}, \mathrm{H}-3 ") / \delta_{\mathrm{C}} 78,3$ $(\mathrm{C}-3 ")$ e $\delta_{\mathrm{H}} 3,86(\mathrm{~m}, \mathrm{H}-4 ") / \delta_{\mathrm{C}} 88,1(\mathrm{C}-4 ")$ confirmaram a presença da unidade de arabinose. Os dados espectroscópicos para a substância $\mathbf{5}$ foram condizentes com os da literatura ${ }^{8}$ para 3-O-arabinofuranosilquercetina (avicularina).

A substância 6 foi obtida da fração clorofórmica dos galhos como um sólido amarelo. A análise dos dados de $\mathrm{RMN}{ }^{1} \mathrm{H}$ e ${ }^{13} \mathrm{C}$ e a comparação com aqueles da literatura ${ }^{9}$ permitiram caracterizar inequivocamente a substância como o isoflavonoide 4',7-di-hidroxi-isoflavona (daidzeína).

A substância $\mathbf{7}$ foi obtida em mistura com o flavonoide $\mathbf{1}$ na proporção de 2:1. Esta mistura foi obtida nesta proporção nas frações acetato de etila das folhas e dos galhos e $n$-butanólica das folhas como sólido amarelo-castanho. O espectro de RMN ${ }^{1} \mathrm{H}$ apresentou 3 sinais na região de hidrogênios aromáticos: $\delta_{\mathrm{H}} 6,80(d, J=8,7 \mathrm{~Hz}$, H-3' e $\left.5^{\prime}\right)$ e 7,29 ( $d, J=8,7 \mathrm{~Hz}, \mathrm{H}-2^{\prime}$ e $\left.6^{\prime}\right)$ característicos de anel $\mathbf{B}$ $p$-hidroxilado, e em $\delta_{\mathrm{H}} 5,95(s l, \mathrm{H}-6)$ correspondente ao anel A. Ainda foram observados sinais de hidrogênios alifáticos de sistema $\mathrm{ABX}$ em $\delta_{\mathrm{H}} 5,33(d d, J=12,3$ e $3,0 \mathrm{~Hz}, \mathrm{H}-2), 2,72(d d, J=17,1$ e $3,0 \mathrm{~Hz}$, $\left.\mathrm{H}_{\mathrm{a}}-3\right)$ e $3,11\left(d d, J=17,1\right.$ e $\left.12,3 \mathrm{~Hz}, \mathrm{H}_{\mathrm{b}}-3\right)$ caracterizando o anel C do esqueleto flavanona. As constantes de acoplamento observadas para H-2 indicaram interação axial-axial e, consequentemente, uma conformação de H-2 em axial. Além destes, foram observados sinais adicionais característicos de uma unidade glicosídica, porém a ausência de sinal referente a hidrogênio anomérico sugeriu uma ligação C-glicosídica. O espectro de $\mathrm{RMN}{ }^{13} \mathrm{C}$ apresentou 21 carbonos, sendo 12 carbonos aromáticos, 5 carbonos oximetínicos, 2 carbonos metilênicos e 1 carbono metínico. A posição da unidade $C$-glicosilada no C-8 foi confirmada pelas interações heteronucleares do $\mathrm{H}-1$ " $\left(\delta_{\mathrm{H}} 4,78\right)$ $\operatorname{com} \delta_{\mathrm{C}} 106,0(\mathrm{C}-8)$ e do H-6 $\left(\delta_{\mathrm{H}} 5,95\right)$ com os sinais $\delta_{\mathrm{C}} 164,2(\mathrm{C}-5) \mathrm{e}$ 167,5 (C-7), observadas pelo mapa de contornos HMBC. Através dos dados espectroscópicos uni- e bidimensionais e comparação destes com os encontrados na literatura, ${ }^{9}$ esta substância foi caracterizada como sendo 8-C- $\beta$-D-glicopiranosil-4',5,7-tri-hidroxiflavanona (iso-hemifloina).

A substância 8 foi isolada da fração acetato de etila das folhas como um sólido amorfo amarelo com rotação específica $[\alpha]_{\mathrm{D}}^{20}=$ $+55,26\left(\mathrm{C}=3,8 \mathrm{mg} / \mathrm{mL}\right.$, acetona). Nos espectros de $\mathrm{RMN}{ }^{1} \mathrm{H} \mathrm{e}{ }^{13} \mathrm{C}$ foram observados sinais com deslocamentos químicos característicos para duas unidades de epicatequina ligadas entre si. A presença de um sistema $\mathrm{AB}$ entre os sinais $\delta_{\mathrm{H}} 4,05(1 \mathrm{H}, d, J=3,3 \mathrm{~Hz}, \mathrm{H}-3) \mathrm{e}$ $4,40(1 \mathrm{H}, d, J=3,3 \mathrm{~Hz}, \mathrm{H}-4)$, típico de hidrogênios de anel $\mathrm{C}$ de uma procianidina do tipo $\mathrm{A}$, onde as unidades de flavonois estão ligadas através de 2 ligações (C-C e C-O). O deslocamento em $\delta_{\mathrm{C}} 100,3$ do C-2 da epicatequina, devido à ligação deste com o oxigênio da outra unidade, é típico de ligação $\mathrm{C}-2_{\mathrm{C}} \rightarrow \mathrm{O} \rightarrow \mathrm{C}-7_{\mathrm{D}}$. Através do mapa de contornos HMBC foi possível observar as interações heteronuclear entre o H-6 ${ }_{\mathrm{D}}^{\prime}$ com os carbonos C-5' e C-7' e, ainda, a interação do $\mathrm{H}-4_{\mathrm{C}}$ com os carbonos C- $8_{\mathrm{D}}^{\prime}$ e C-8' $\mathrm{a}_{\mathrm{F}}$, que comprovaram as ligações $\mathrm{C}-4_{\mathrm{C}} \rightarrow \mathrm{C}-8_{\mathrm{D}}^{\prime}{ }_{\mathrm{D}}$ e C-2 ${ }_{\mathrm{C}} \rightarrow \mathrm{C}-7_{\mathrm{D}}{ }_{\mathrm{D}}$ entre os flavonoides. A configuração relativa 2',3'-cis no anel $\mathrm{F}$ foi atribuída pela observação do sinal de H-2' (em $\left.\delta_{\mathrm{H}} 4,91\right)$ como um singleto. A rotação específica observada para 8 e os dados de $\mathrm{RMN}{ }^{1} \mathrm{H} \mathrm{e}{ }^{13} \mathrm{C}$ foram concordantes com os da literatura ${ }^{10}$ para a epicatequina- $\left(4 \beta \rightarrow 8^{\prime}, 2 \beta \rightarrow O \rightarrow 7^{\prime}\right)$-epicatequina (procianidina A2).

A substância 9 foi obtida das frações aquosas das folhas e dos galhos como um sólido amarelo. $\mathrm{O}$ espectro de $\mathrm{RMN}{ }^{1} \mathrm{H}$ de 9 apresentou um singleto em $\delta_{\mathrm{H}} 3,60$, referente a hidrogênios oximetílicos, e sinais de hidrogênios metínicos em $\delta_{\mathrm{H}} 3,88(d, J=2,4 \mathrm{~Hz}$; H-1 e 6), 3,74 ( $d d, J=9,9$ e 2,4 Hz; H-2), 3,69 ( $d d, J=9,9$ e 2,4 Hz; H-5), 3, 25 $(d d, J=9,6$ e $9,0 \mathrm{~Hz} ; \mathrm{H}-3)$ e 3,59 ( $t, J=9,9 \mathrm{~Hz} ; \mathrm{H}-4)$. Nos espectros de RMN ${ }^{13} \mathrm{C} / \mathrm{DEPT}$ foram observados 7 sinais, sendo 6 de carbonos metínicos em $\delta_{\mathrm{C}} 84,8(\mathrm{C}-3), 74,2$ (C-4), 73,7 (C-6), 73,4 (C-1), 72,5 (C-5), 72,0 (C-2) e um de carbono metílico em $\delta_{\mathrm{C}} 60,8$. A posição do grupo metoxila em C-3 foi confirmada pela correlação do H-3 $\left(\delta_{\mathrm{C}}\right.$ $3,25)$ com o C-7 $\left(\mathrm{OCH}_{3}\right)$ e dos hidrogênios metílicos $\left(\delta_{\mathrm{C}} 3,60\right)$ com o C-3 $\left(\delta_{\mathrm{C}} 84,8\right)$ pelo mapa de contornos HMBC. Estes dados foram concordantes com os da literatura ${ }^{11}$ para o poliol 3-O-metil-quiro-inositol.

Ensaios de atividade anti-inflamatória foram realizados para os extratos brutos das folhas e dos galhos e suas frações. Foi utilizado o modelo de edema de orelha induzido por óleo de cróton em camundongo, na administração da dose de 5,0 mg/orelha, por via tópica. Nestes ensaios todas as amostras inibiram o edema de orelha, sendo que os extratos bruto e das frações das folhas apresentaram inibição de 54, 57, 30, 55, 35 e 46\% para o extrato EBF e as frações FHF, FCF, FAEF, FBF e FAF; os extratos dos galhos, EBG e suas frações 
FHG, FCG, FAEG, FBG e FAG, inibiram em 52, 77, 8, 26, 31 e 6\%.

A atividade sobre a enzima mieloperoxidase (MPO), utilizada como indicativo da presença de leucócitos polimorfonucleares, foi também avaliada para os extratos brutos e as frações polares de $A$. fluminensis. A MPO é uma heme-enzima considerada responsável pela resposta imunológica inespecífica a vários agentes. ${ }^{12}$ Os resultados mostraram que a administração na dose de $5,0 \mathrm{mg} /$ orelha inibiu a atividade sobre a enzima em 0,$3 ; 17 ; 61,7 ; 67,1 ; 86,4 ; 78,8 ; 77,0$ e $50,9 \%$ nos extratos EBF e EBG e as frações FAEF, FAEG, FBF, FBG, FAF e FAG, respectivamente. O processo anti-inflamatório tópico pode ser promovido por diferentes mediadores na formação do edema. A maior atividade observada na enzima mieloperoxidase indica a migração de células polimorfonucleares para o local inflamado.

A canferitrina (1) está presente nas frações polares (FAE e FB) das folhas e dos galhos em quantidades expressivas. Esta substância e também a quercitrina (4) e a procianidina $\mathrm{A}_{2}(\mathbf{8})$, isoladas da FAEF, são conhecidas como agentes anti-inflamatórios e a presença das mesmas contribui para as significativas atividades observadas nestas frações. ${ }^{13}$

\section{CONCLUSÃO}

O estudo fitoquímico das folhas e dos galhos de A. fluminensis resultou no isolamento dos flavonoides canferitrina, $\alpha$-raminoisorobina, 3-O-apiofuranosil-7- $O$-raminopiranosilcanferol, quercitrina, avicularina, daidzeina, iso-hemifloina, da procianidina $\mathrm{A}_{2}$, do poliol 3-O-metil-quiro-inositol e dos esteroides sitosterol e estigmasterol. Este é o primeiro relato do isolamento destas substâncias no gênero Aeschynomene. Os extratos brutos das folhas e dos galhos e frações provenientes destes extratos apresentaram significativa atividade anti-inflamatória para o modelo de edema de orelha e MPO.

\section{MATERIAL SUPLEMENTAR}

Está disponível em http://quimicanova.sbq.org.br, em arquivo pdf, com acesso livre.

\section{AGRADECIMENTOS}

À Fundação Araucária, PIQDT - CAPES e UEM pelo apoio financeiro para o desenvolvimento deste trabalho.

\section{REFERÊNCIAS}

1. Fernandes, A.; O taxon Aeschynomene no Brasil, EUFC: Fortaleza, 1996.

2. Braggio, M. M.; Lima, M. E. L.; Veasey, E. A.; Haraguchi, M.; Arq. Inst. Biol. 2002, 69, 49; Fullas, F.; Kornberg, L. J.; Wani, M. C.; Wall, M. E.; Farnsworth, N.; Chagwedera, T. E.; Kinghorn, A. D.; J. Nat. Prod. 1996, 59, 190; Powell, R. G.; Smith-Jr., C. R.; Weisleder, D.; Phytochemistry 1984, 23, 2789; Ueda, M.; Hiraoka, T.; Niwa, M.; Yamamura, S.; Tetrahedron Lett. 1999, 40, 6777; Ueda, M.; Niwa, M.; Yamamura, S.; Phytochemistry 1995, 39, 817.

3. van Arman, G. C.; Clin. Pharmacol. Ther. 1974, 16, 900.

4. Bradley, P. P.; Priebat, D. A.; Chiristensen, R. D.; Rothstein, G.; J. Invest. Dermatol. 1982, 78, 206.

5. Aragão, P. C. A.; Toledo, J. B.; Morais, A. A.; Braz-Filho, R.; Quim. Nova 1990, 13, 254

6. Chua, M.-T.; Tung, Y.-T.; Chang, S.-T.; Bioresour. Technol. 2008, 99, 1918; Spanou, C.; Bourou, G.; Dervishi, A.; Aligiannis, N.; Angelis, A.; Komiotis, D.; Skaltsounis, A.-L.; Kouretas, D.; J. Agric. Food Chem. 2008, 56, 6967.

7. Sirat, H. M.; Rezali, M. F.; Ujang, Z.; J. Agric. Food Chem. 2010, 58, 10404.

8. Velozo, L. S. M.; Da Silva, B. P.; Da Silva, E, M. B.; Parente, J. P.; Fitoterapia 1999, 70, 532.

9. Agrawal, P. K.; Studies in Organic Chemistry: Carbon-13 NMR of Flavonoids, Ed. Elsevier Science: Lucknow, 1989.

10. Lou, H.; Yamazaki, Y.; Sasaki, T.; Uchida, M.; Tanaka, H.; Oka, S.; Phytochemistry 1999, 51, 297.

11. DellaGreca, M.; Fiorentino, A.; Izzo, A.; Napoli, F.; Purcaro, R.; Zarrelli, A.; Chem. Biodiversity 2007, 4, 118.

12. Regasini, L. O.; Fernandes, D. C.; Castro-Gamboa, I.; Silva, D. H. S.; Furlan, M.; Barreiro, E. J.; Cardoso-Lopes, E. M.; Young, C. M.; Torres, L. B.; Vellosa, J. C. R.; Oliveira, O. M. M.; Bolzani, V. S.; Quim. Nova 2008, 31, 802 .

13. Pizzollatti, M. G.; Cunha Jr., A.; Szpoganicz, B.; Sousa, E.; Quim. Nova 2003, 26, 466; Toker, G.; K peli, E.; Memisoglu, M.; Yesilada, E.; J. Ethnopharmacol. 2004, 95, 393; Yen, C.-T.; Hsieh, P.-W.; Hwang, T.-L.; Lan, Y.-H.; Chang, F.-R.; Wu, Y.-C.; Chem. Pharm. Bull. 2009, 57, 280; Nandakumar, V.; Singh, T.; Katiyar, S.; Cancer Lett. 2008, 269, 378. 
ESTUDO FITOQUÍMICO E AVALIAÇÃO DA ATIVIDADE ANTI-INFLAMATÓRIA DE Aeschynomene fluminensis VELL. (FABACEAE)

Marlene Capelin Ignoato, Rodrigo Monteiro Fabrão, Ivânia Teresinha Albrecht Schuquel, Marcos Felipe Pinatto Botelho e Silvana Maria de Oliveira Santin*

Departamento de Química, Universidade Estadual de Maringá, 87020-900 Maringá - PR, Brasil

Laura Lícia Milani de Arruda e Ciomar Aparecida Bersani-Amado

Departamento de Farmacologia e Terapêutica, Universidade Estadual de Maringá, 87020-900 Maringá - PR, Brasil

Maria Conceição de Souza

Departamento de Biologia, Universidade Estadual de Maringá, 87020-900 Maringá - PR, Brasil

Tabela 1S. Dados de RMN de ${ }^{13} \mathrm{C} / \mathrm{DEPT}(75,45 \mathrm{MHz})$ e ${ }^{1} \mathrm{H}(300,06 \mathrm{MHz})$ e gHMBC para a substância 7 em CD $\mathrm{OD}_{3}$ [ppm] (multiplicidade $J \mathrm{~Hz}$ ) e dados da literatura9 para a iso-hemifloina

\begin{tabular}{|c|c|c|c|}
\hline $\mathrm{C}$ & 7 & ${ }^{1} \mathrm{H} / \mathrm{gHSQC}$ & iso-hemifloina $^{9}$ \\
\hline 2 & $80,4(\mathrm{CH})$ & $5,33 d d / \mathrm{J}=3,0$ e $12,3 \mathrm{~Hz}$ & $78,4(\mathrm{CH})$ \\
\hline 3 & $43,9\left(\mathrm{CH}_{2}\right)$ & $\begin{array}{l}\mathrm{Ha} 2,72 d d / \mathrm{J}=3,0 \text { e } 17,1 \mathrm{~Hz} \\
\mathrm{Hb} 3,11 d d / \mathrm{J}=12,3 \text { e } 17,1 \mathrm{~Hz}\end{array}$ & $42,1\left(\mathrm{CH}_{2}\right)$ \\
\hline 4 & $198,0(\mathrm{C})$ & & $196,6(C)$ \\
\hline 5 & $164,2(\mathrm{C})$ & & $161,8(\mathrm{C})$ \\
\hline 6 & $96,4(\mathrm{CH})$ & $5,95 s l$ & $95,0(\mathrm{C})$ \\
\hline 7 & $167,5(\mathrm{C})$ & & $166,1(\mathrm{C})$ \\
\hline 8 & $106,0(\mathrm{C})$ & & $105,9(\mathrm{CH})$ \\
\hline $9(8 a)$ & $163,0(\mathrm{C})$ & & $162,9(\mathrm{C})$ \\
\hline $10(4 a)$ & $103,2(\mathrm{C})$ & & $101,6(\mathrm{C})$ \\
\hline $1^{\prime}$ & $130,9(\mathrm{C})$ & & $129,0(\mathrm{C})$ \\
\hline 2' e 6' & $129,0(\mathrm{CH})$ & $7,29 \mathrm{~d} / \mathrm{J}=8,7 \mathrm{~Hz}$ & $128,4(\mathrm{CH})$ \\
\hline 3' e 5' & $116,3(\mathrm{CH})$ & $6,80 \mathrm{~d} / \mathrm{J}=8,7 \mathrm{~Hz}$ & $115,4(\mathrm{CH})$ \\
\hline $4^{\prime}$ & $159,0(\mathrm{C})$ & & $157,8(\mathrm{C})$ \\
\hline $1 "$ & $75,2(\mathrm{CH})$ & $4,78 \mathrm{~d} / \mathrm{J}=10,2 \mathrm{~Hz}$ & $73,1(\mathrm{CH})$ \\
\hline $2 "$ & $72,6(\mathrm{CH})$ & $4,12 m$ & $70,5(\mathrm{CH})$ \\
\hline $3 "$ & $80,2(\mathrm{CH})$ & $3,39-3,44 m$ & $79,2(\mathrm{CH})$ \\
\hline $4 "$ & $71,8(\mathrm{CH})$ & $3,32-3,37 \mathrm{~m}$ & $70,8(\mathrm{CH})$ \\
\hline $5 "$ & $82,5(\mathrm{CH})$ & $3,39-3,44 m$ & $81,6(\mathrm{CH})$ \\
\hline \multirow[t]{2}{*}{$6 "$} & $62,9\left(\mathrm{CH}_{2}\right)$ & $\begin{array}{l}\text { Ha } 3,71 d d / \mathrm{J}=2,7 \text { e } 5,4 \mathrm{~Hz} \\
\text { Hb } 3,82 d d / \mathrm{J}=2,7 \text { e } 5,4 \mathrm{~Hz}\end{array}$ & $61,7\left(\mathrm{CH}_{2}\right)$ \\
\hline & & & $\left(\mathrm{DMSO}_{\mathrm{d}}\right)$ \\
\hline
\end{tabular}

*e-mail: smoliveira@uem.br

\#Artigo em homenagem ao Prof. Otto R. Gottlieb (31/8/1920-19/6/2011) 
Tabela 2S. Dados de RMN de ${ }^{13} \mathrm{C} / \mathrm{DEPT}(75,5 \mathrm{MHz})$ e ${ }^{1} \mathrm{H}(300 \mathrm{MHz})$ para a substância 8 em $\mathrm{CD}_{3} \mathrm{OD}$ [ppm] (multiplicidade $J \mathrm{~Hz}$ ) e dados da literatura ${ }^{10}$ para o dímero

\begin{tabular}{|c|c|c|c|c|}
\hline $\mathrm{C}$ & 8 & ${ }^{1} \mathrm{H} / \mathrm{HSQC}$ & Procianidina $\mathrm{A}_{2} /{ }^{1} \mathrm{H}$ & \\
\hline 2 & $100,3(\mathrm{C})$ & & 100,2 & \\
\hline 3 & $68,2(\mathrm{CH})$ & $4,05 d / J=3,3 \mathrm{~Hz}$ & 68,1 & $4,05 \mathrm{~d} / \mathrm{J}=3,4 \mathrm{~Hz}$ \\
\hline 4 & $29,4(\mathrm{CH})$ & $4,40 \mathrm{~d} / \mathrm{J}=3,3 \mathrm{~Hz}$ & 29,3 & $4,39 \mathrm{~d} / \mathrm{J}=3,4 \mathrm{~Hz}$ \\
\hline $4 a$ & $104,4(\mathrm{C})$ & & 104,3 & \\
\hline 5 & $157,2(\mathrm{C})$ & & 157,1 & \\
\hline 6 & $98,4(\mathrm{CH})$ & $6,00 \mathrm{~d} / \mathrm{J}=2,4 \mathrm{~Hz}$ & 98,3 & $6,00 \mathrm{~d} / \mathrm{J}=2,3 \mathrm{~Hz}$ \\
\hline 7 & $158,3(\mathrm{C})$ & & 158,2 & \\
\hline 8 & $96,8(\mathrm{CH})$ & $6,06 \mathrm{~d} / \mathrm{J}=2,4 \mathrm{~Hz}$ & 96,7 & $6,07 \mathrm{~d} / \mathrm{J}=2,3 \mathrm{~Hz}$ \\
\hline $8 a$ & $154,4(\mathrm{C})$ & & 154,3 & \\
\hline 9 & $132,6(\mathrm{C})$ & & 132,5 & \\
\hline 10 & $115,8(\mathrm{CH})$ & $7,14 d / \mathrm{J}=2,1 \mathrm{~Hz}$ & 115,7 & $7,14 \mathrm{~d} / \mathrm{J}=2,4 \mathrm{~Hz}$ \\
\hline 11 & $146,5(\mathrm{C})$ & & 146,3 & \\
\hline 12 & $145,8(\mathrm{C})$ & & 145,7 & \\
\hline 13 & $116,2(\mathrm{CH})$ & $6,81 \mathrm{~d} / \mathrm{J}=8,1 \mathrm{~Hz}$ & 116,1 & $6,82 \mathrm{~d} / \mathrm{J}=8,2 \mathrm{~Hz}$ \\
\hline 14 & $120,5(\mathrm{CH})$ & $7,02 d d / \mathrm{J}=1,8$ e $3,9 \mathrm{~Hz}$ & 120,4 & $7,07 d d / \mathrm{J}=2,4$ e $8,2 \mathrm{~Hz}$ \\
\hline $2^{\prime}$ & $81,9(\mathrm{CH})$ & 4,91 br.s & 81,8 & 4,91 br.s \\
\hline $3^{\prime}$ & $67,2(\mathrm{CH})$ & $4,23 d / J=3,3 \mathrm{~Hz}$ & 67,0 & 4,23 br.s \\
\hline \multirow[t]{3}{*}{4 ' } & $30,1\left(\mathrm{CH}_{2}\right)$ & $\mathrm{H} \alpha 2,75 d d / \mathrm{J}=2,4$ e $17,1 \mathrm{~Hz}$ & & \\
\hline & & $\mathrm{H} \beta 2,94 d d / \mathrm{J}=2,4$ e $17,3 \mathrm{~Hz}$ & 29,9 & $\mathrm{H} \alpha 2,74 d d / \mathrm{J}=3,2$ e $17,3 \mathrm{~Hz}$ \\
\hline & & & & $\mathrm{H} \beta 2,95 d d / \mathrm{J}=4,6$ e $17,3 \mathrm{~Hz}$ \\
\hline 4'a & $102,6(\mathrm{C})$ & & 102,5 & \\
\hline 5 , & $156,8(\mathrm{C})$ & & 156,7 & \\
\hline $6{ }^{\prime}$ & $96,6(\mathrm{CH})$ & $6,08 s l$ & 96,5 & $6,09 s$ \\
\hline 7' & $152,5(\mathrm{C})$ & & 152,3 & \\
\hline $8^{\prime}$ & $107,4(\mathrm{C})$ & & 107,3 & \\
\hline 8 'a & $152,3(\mathrm{C})$ & & 152,3 & \\
\hline 9' & $131,4(\mathrm{C})$ & & 131,2 & \\
\hline $10^{\prime}$ & $115,8(\mathrm{CH})$ & $7,12 \mathrm{~d} / \mathrm{J}=2,1 \mathrm{~Hz}$ & 115,7 & $7,13 d / \mathrm{J}=2,2 \mathrm{~Hz}$ \\
\hline $11^{\prime}$ & $146,9(\mathrm{C})$ & & 146,8 & \\
\hline $12^{\prime}$ & $146,2(\mathrm{C})$ & & 146,0 & \\
\hline $13{ }^{\prime}$ & $116,1(\mathrm{CH})$ & $6,79 \mathrm{~d} / \mathrm{J}=8,1 \mathrm{~Hz}$ & 116,0 & $6,81 \mathrm{~d} / \mathrm{J}=8,2 \mathrm{~Hz}$ \\
\hline \multirow[t]{2}{*}{$14^{\prime}$} & $119,9(\mathrm{CH})$ & $6,99 d d / \mathrm{J}=1,5$ e $3,9 \mathrm{~Hz}$ & 119,8 & $6,99 d d / \mathrm{J}=2,2$ e $8,2 \mathrm{~Hz}$ \\
\hline & & & $\mathrm{CD}_{3} \mathrm{OD}$ & \\
\hline
\end{tabular}

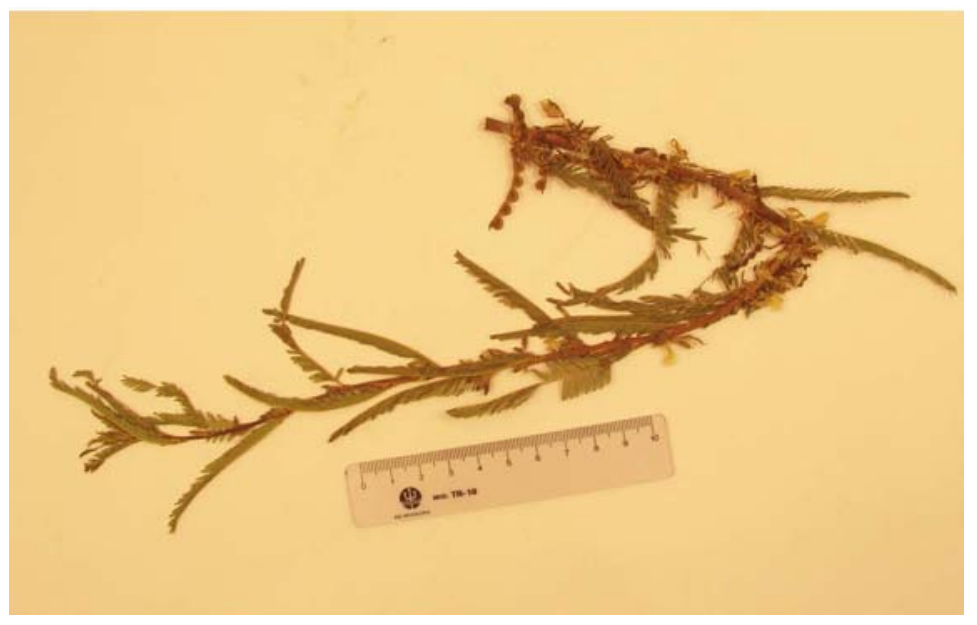

Figura 1S. Aeschynomene fluminensis - foto cedida pelo Laboratório de Mata Ciliar/Nupélia/UEM 


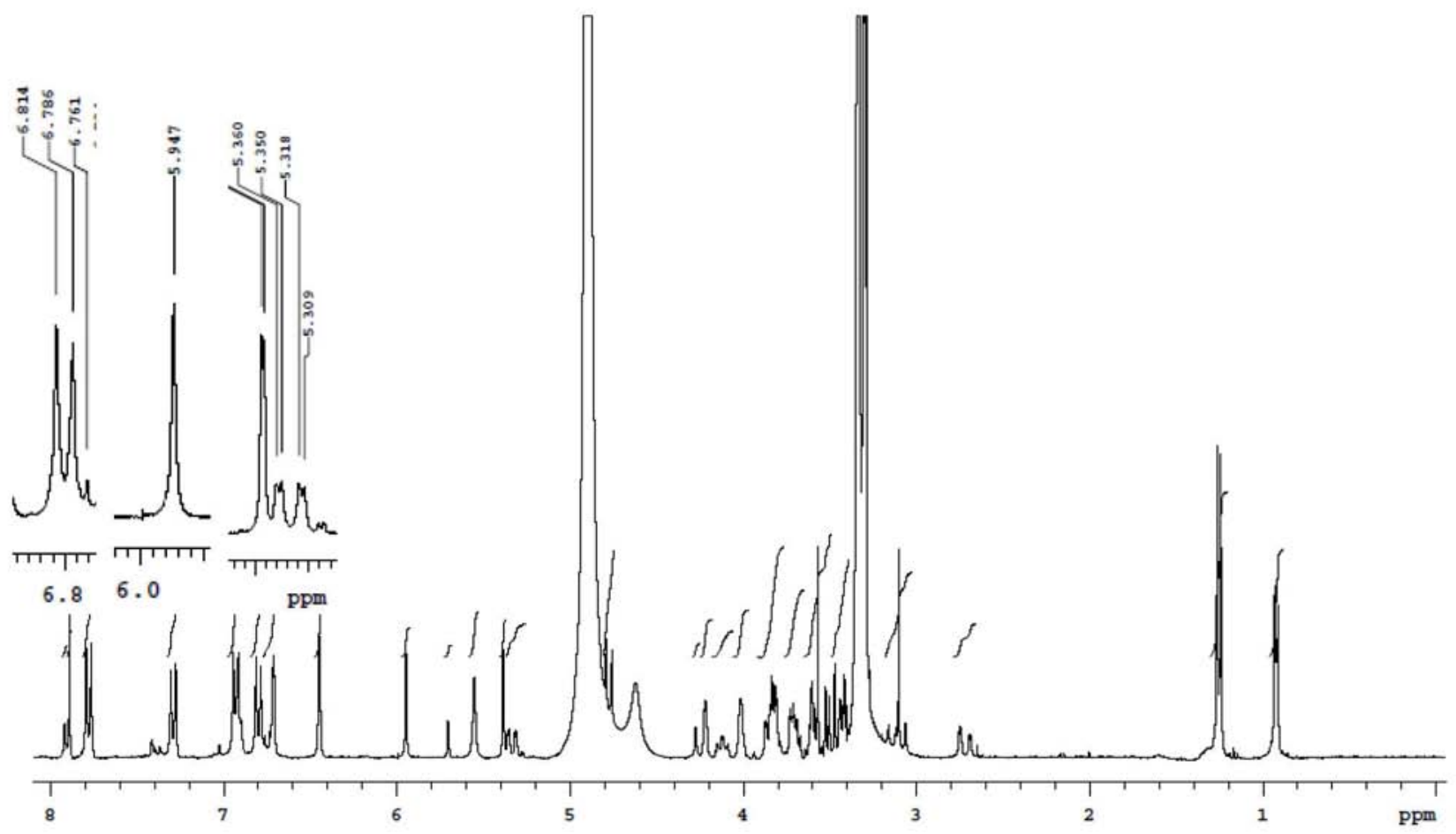

Figura 2S. Espectro de $\mathrm{RMN} \mathrm{de}^{1} \mathrm{H}\left(\mathrm{CD}_{3} \mathrm{OD} ; 300 \mathrm{MHz}\right)$ para a mistura 1 e 7

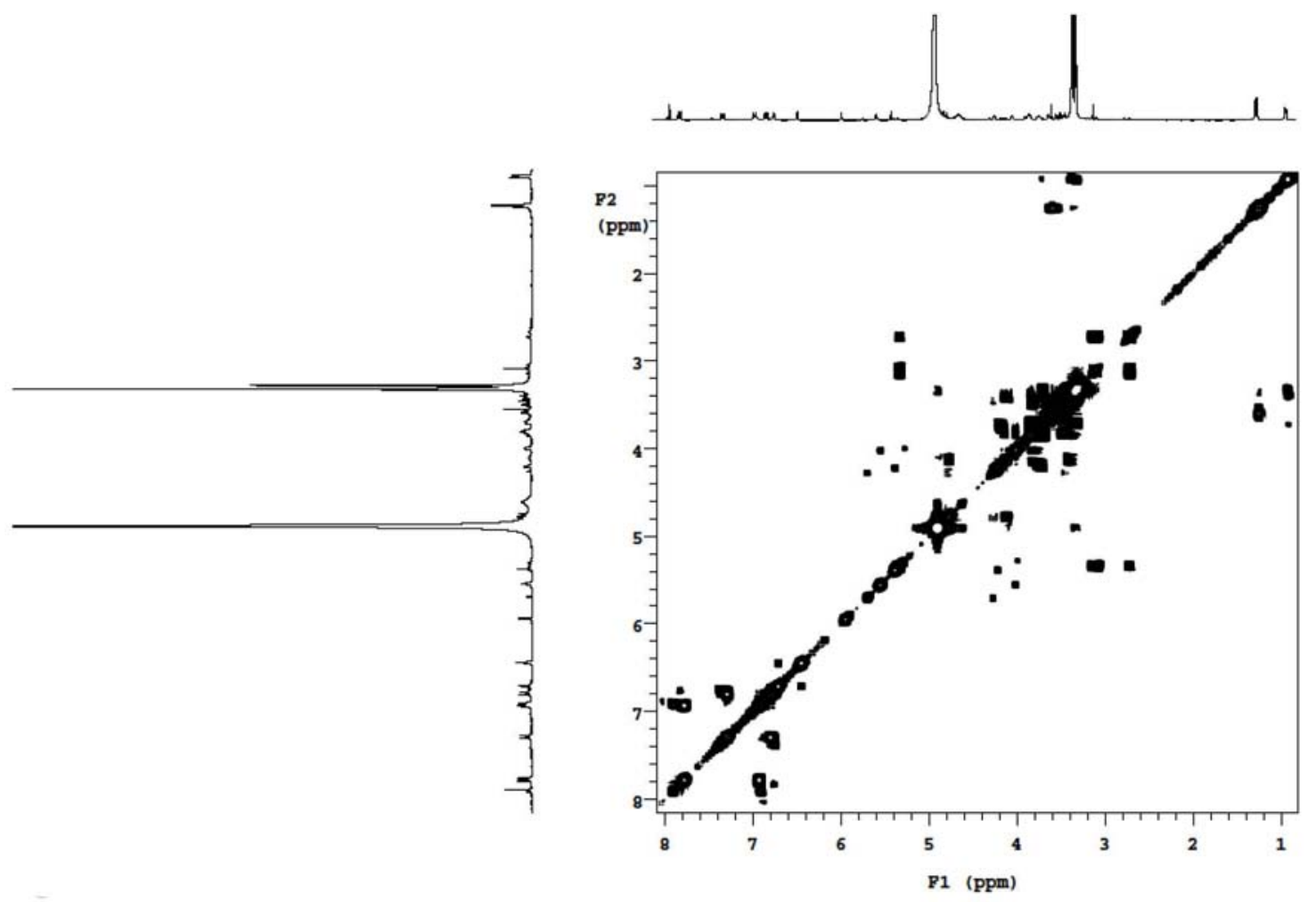

Figura 3S. Mapa de contornos gCOSY $\left(\mathrm{CD}_{3} \mathrm{OD} ; 300 \mathrm{MHz}\right)$ para a mistura das substâncias 1 e 7 


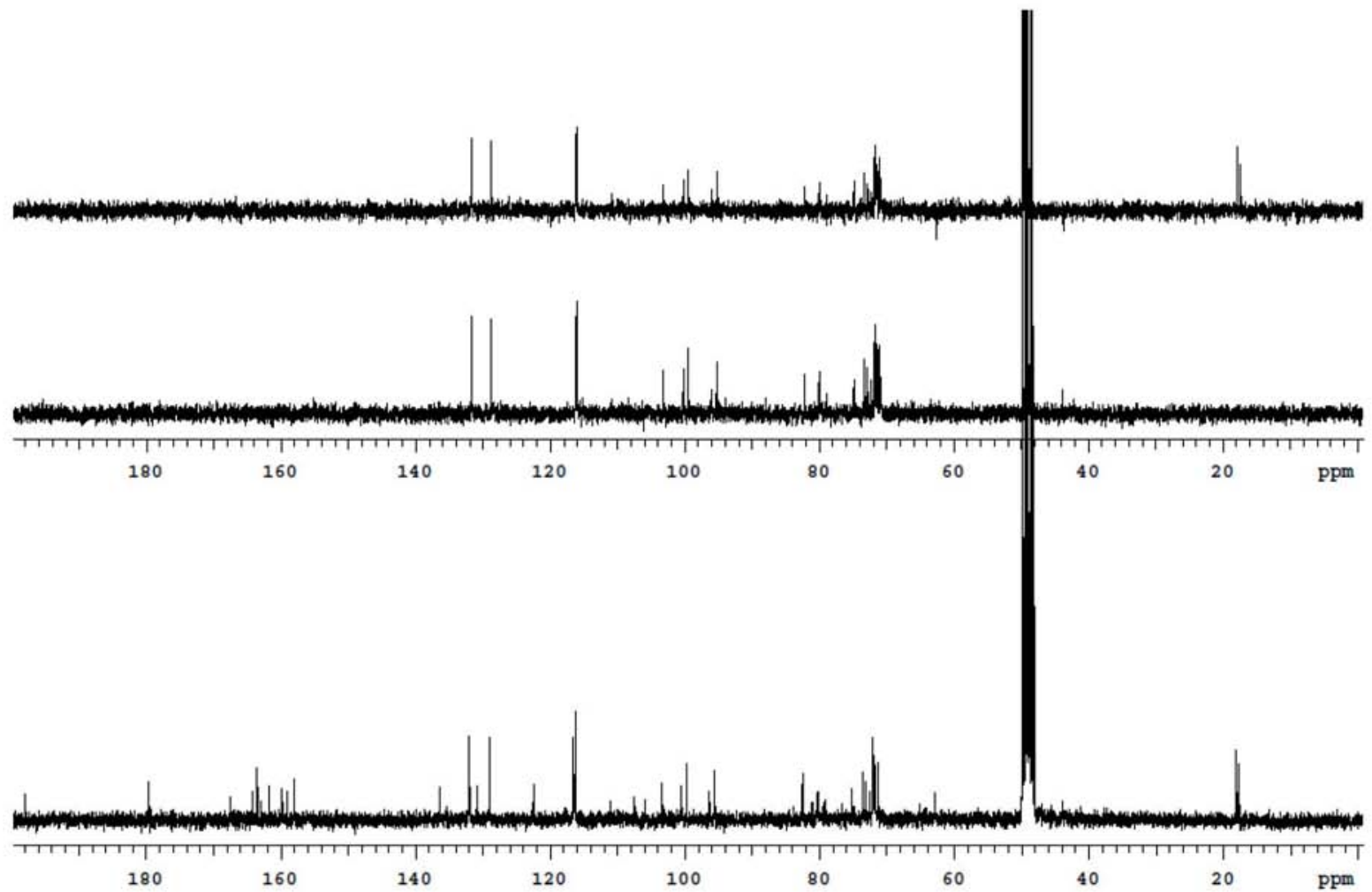

Figura 4S. Espectro de RMN de ${ }^{13} \mathrm{C} / D E P T\left(C D_{3} O D ; 75,5 \mathrm{MHz}\right)$ para a mistura das substâncias 1 e 7

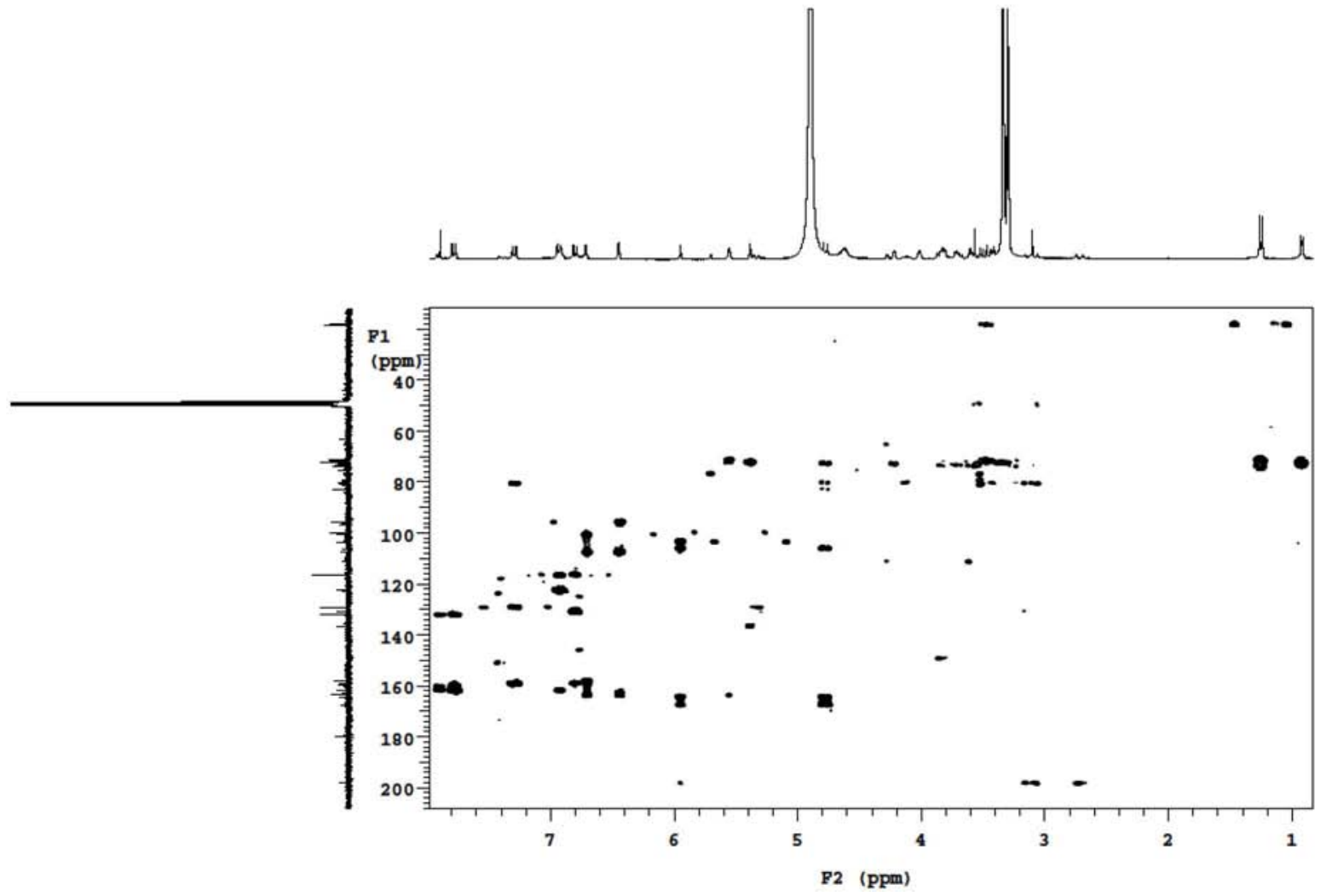

Figura 5S. Mapa de contornos $g \mathrm{HMBC}\left(C D_{3} \mathrm{OD} ; 75,5\right.$ e $\left.300 \mathrm{MHz}\right)$ para a mistura das substâncias 1 e 7 


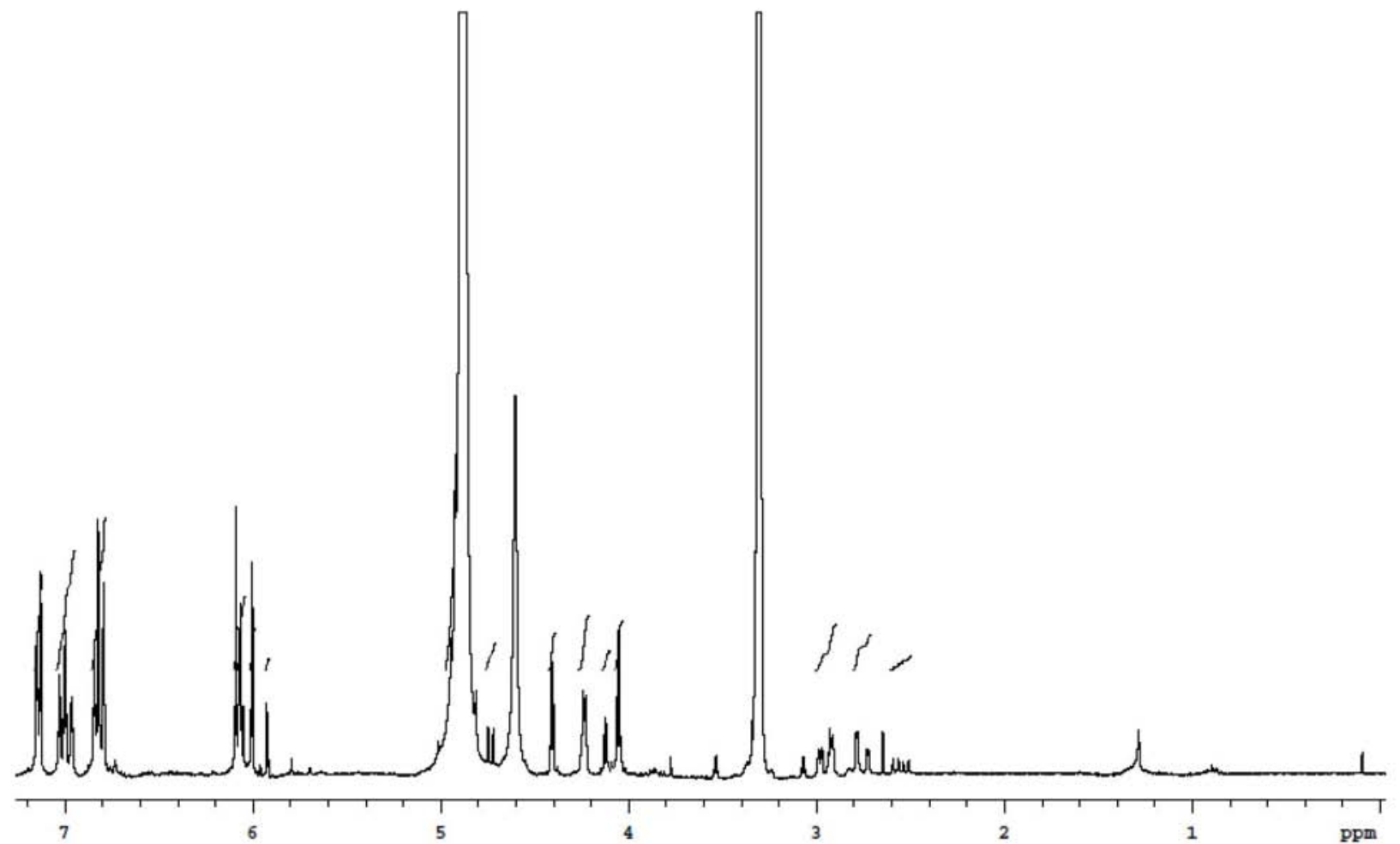

Figura 6S. Espectro de $\mathrm{RMN} \mathrm{de}{ }^{1} \mathrm{H}\left(\mathrm{CD}_{3} \mathrm{OD} ; 300 \mathrm{MHz}\right)$ para a substância 8

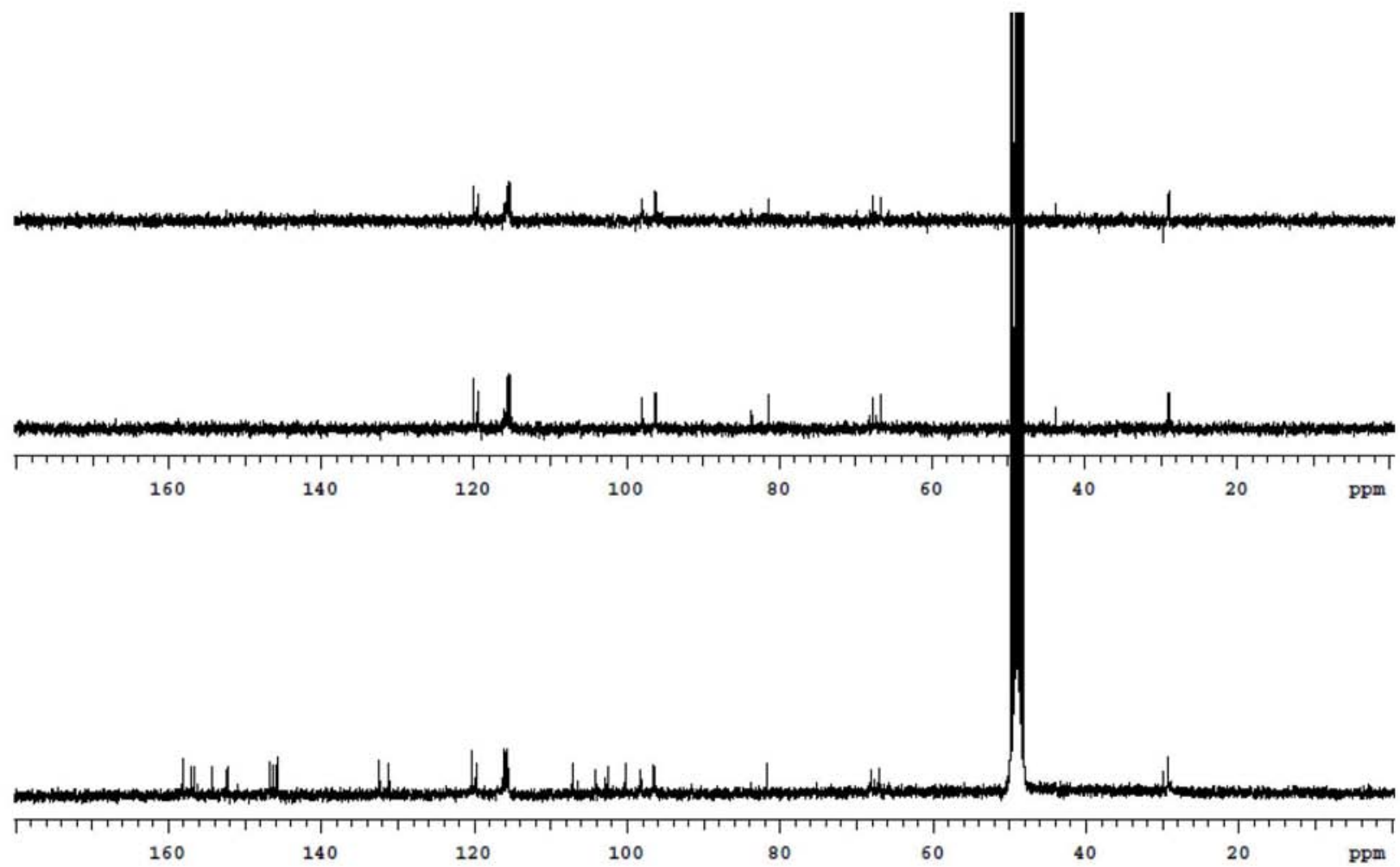

Figura 7S. Espectro de $\mathrm{RMN} d \mathrm{de}^{13} \mathrm{C} / \mathrm{DEPT}\left(\mathrm{CD}_{3} \mathrm{OD} ; 75 \mathrm{MHz}\right)$ para a substância 8 

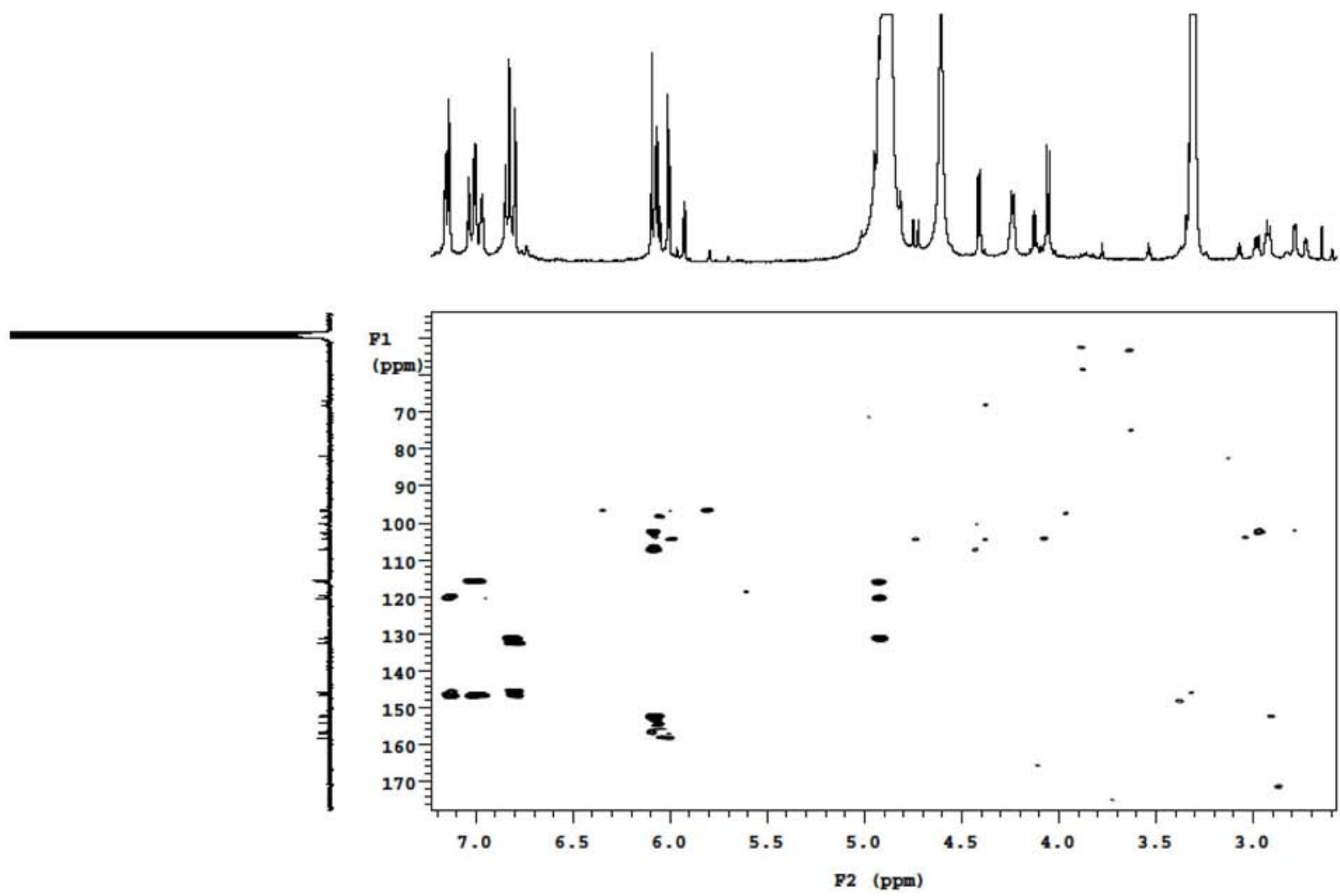

Figura 8S. Mapa de contornos gHMBC (CD 3 OD; 75 e $300 \mathrm{MHz})$ para a substância 8
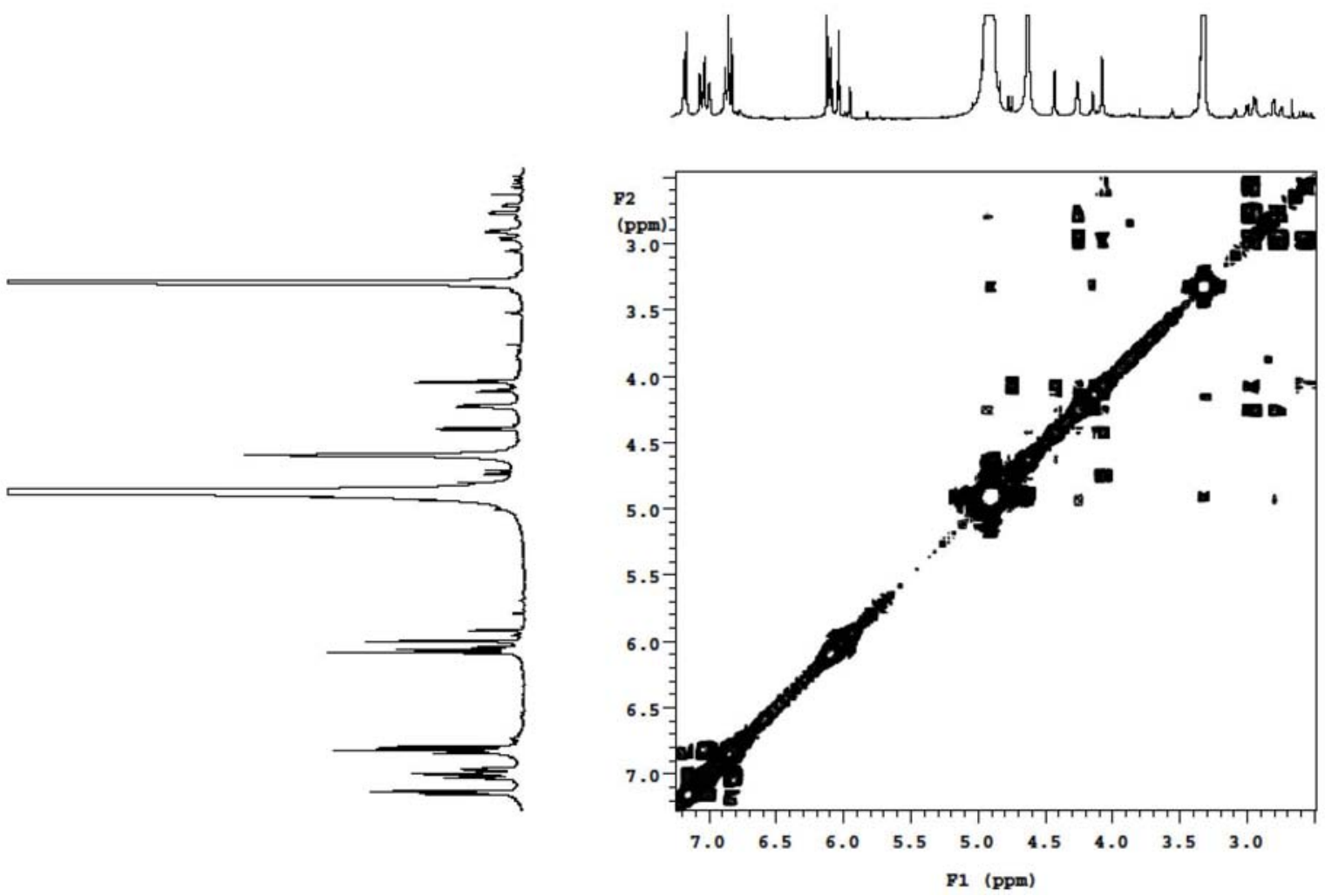

Figura 9S. Mapa de contornos $\mathrm{g} \operatorname{COSY}\left(\mathrm{CD}_{3} \mathrm{OD} ; 300 \mathrm{MHz}\right)$ para a substância 8 

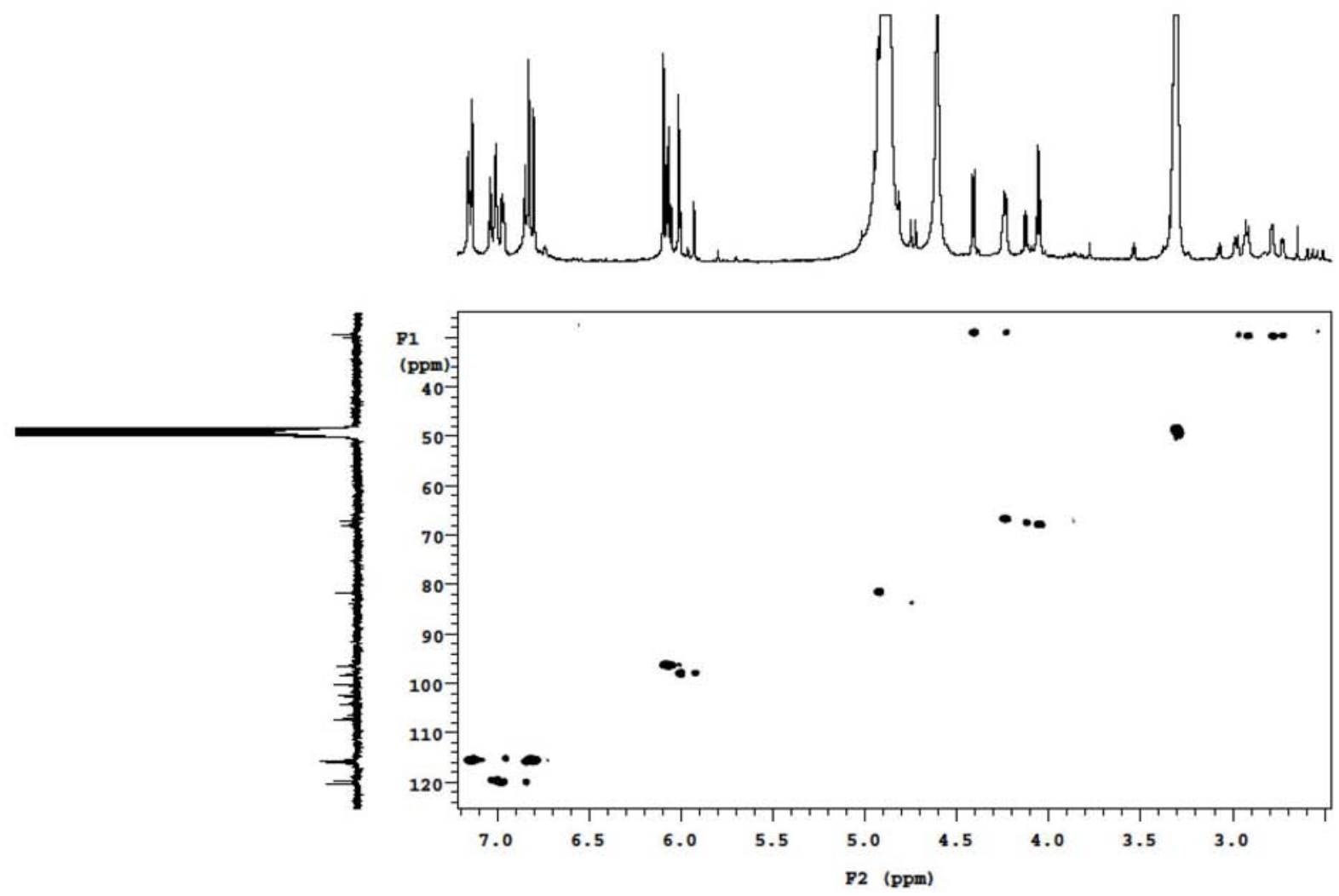

Figura 10S. Mapa de contornos gHSQC $\left(\mathrm{CD}_{3} \mathrm{OD} ; 75\right.$ e $\left.300 \mathrm{MHz}\right)$ para a substância 8
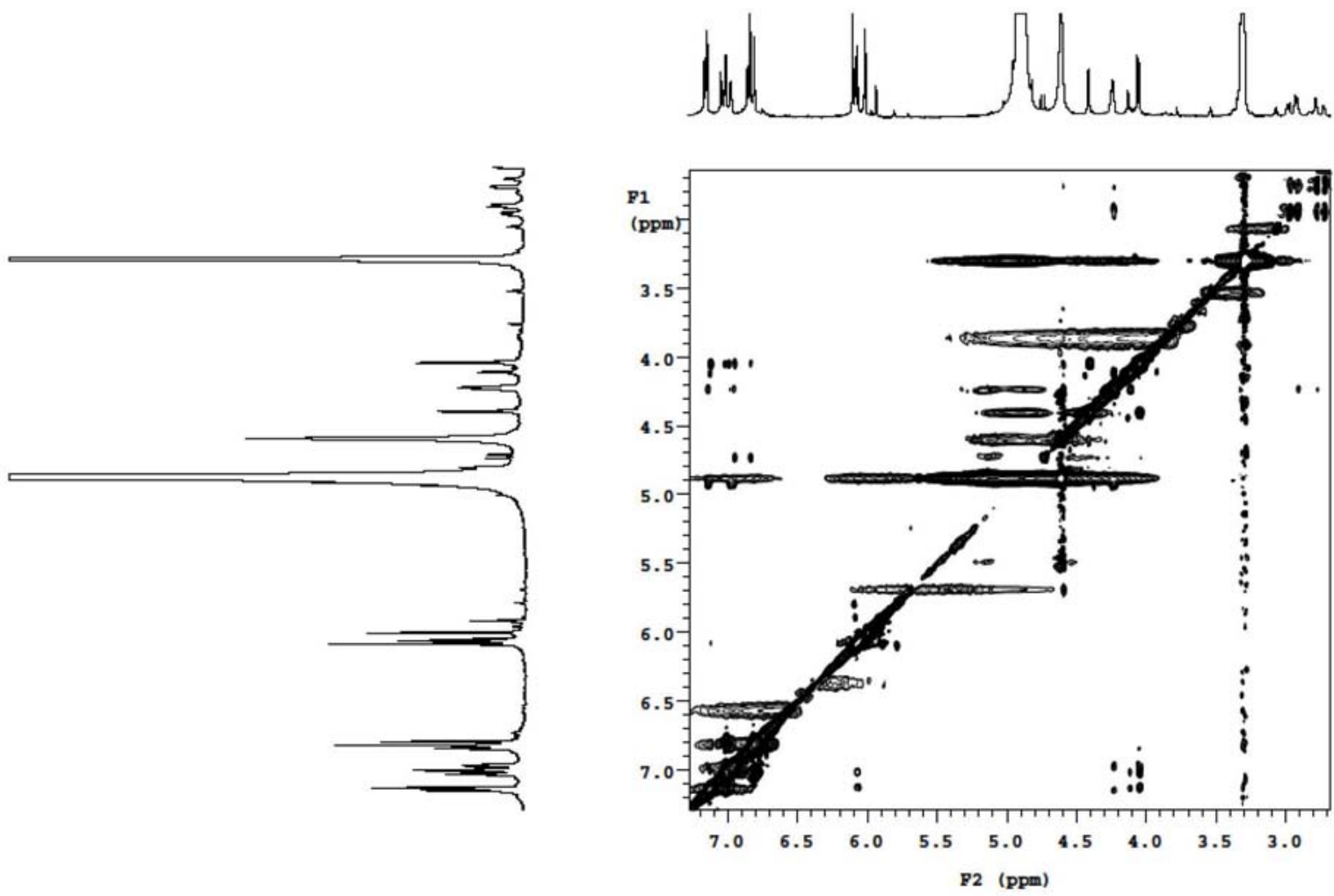

Figura 11S. Mapa de contornos $\mathrm{NOESY}\left(\mathrm{CD}_{3} \mathrm{OD}\right.$; 75 e $\left.300 \mathrm{MHz}\right)$ para a substância 8 